\title{
Multi-RAT for IoT: The Potential in Combining LoRaWAN and NB-IoT
}

\author{
Guus Leenders (D), Gilles Callebaut (D), Geoffrey Ottoy (D), Liesbet Van der Perre (D), Lieven De Strycker (D) \\ KU Leuven, ESAT-DRAMCO, Ghent Technology Campus \\ Ghent, Belgium \\ name.surname@kuleuven.be
}

\begin{abstract}
The broad range of requirements of Internet of Things applications has lead to the development of several dedicated communication technologies, each tailored to meet a specific feature set. A solution combining different wireless technologies in one device, can overcome the disadvantages of any individual technology. The design of such Multiple Radio Access Technology solutions based on the diverse characteristics of the technologies offers interesting opportunities. We have assessed both the potential gains and the overhead that a Multi-RAT solution brings about. To that end, we have evaluated key IoT node requirements in function of payload size and link quality: energy efficiency, coverage, payload size, latency performance, Quality of Service, and cost efficiency. Our assessment and experimental validation of these features show the merits of a Multi-RAT solution. Notably, energy consumption in use cases with only sporadic large payload requirements, can be improved by a factor of at least 4 with respect to either single-mode technologies. Moreover, latency-critical messages can get delivered on time and coverage can be extended elegantly where needed.
\end{abstract}

Index Terms-Internet of Things, Multi-RAT, LoRaWAN, NB-IoT

\section{INTRODUCTION}

The Internet of Things (IoT) is a key technological component in ever more applications. Low Power Wide Area Networks (LPWANs), a subset of IoT connectivity solutions, enable connecting objects over long distances. For example, trees equipped with low-power wireless sensors, allow for remote monitoring of the trees' health, and in a more urban setting, low-power sensors create smart buildings and cities.

When matching wireless connectivity with the requirements of a specific Internet of Things (IoT) application, both technical and non-technical aspects should be considered, as illustrated in Fig. 1. Although each application has its own specific requirements, a large class requires a similar set of features such as long-range wireless connectivity, low energy consumption and cost effectiveness [1] while only transmitting a relatively small amount of data.

Conventional cellular standards $(3 \mathrm{G}, 4 \mathrm{G})$ have been designed to provide global coverage, yet they consume too much energy for battery-powered devices [2]. The introduction of LPWANs has enabled developers to opt for a cost effective low-power connectivity technology, whilst still enabling long-range communication. Long Range Wide Area Network (LoRaWAN) and Narrowband IoT (NB-IoT) are two prominent LPWAN technologies, operating in unlicensed and licensed bands respectively.

LoRaWAN is a network stack that is implemented on top of the Long Range (LoRa) physical layer. It has been rolled out by both commercial operators and non-profit organisations, resulting in wide scale LoRaWAN coverage.

NB-IoT is a cellular LPWAN technology. By reducing the User Equipment (UE)'s complexity and deploying a dedicated modulation inherited from Long Term Evolution (LTE), its battery life and coverage are greatly extended, at the cost of reduced bandwidth and downlink modes. In contrast to traditional cellular transmission, NB-IoT is optimized for

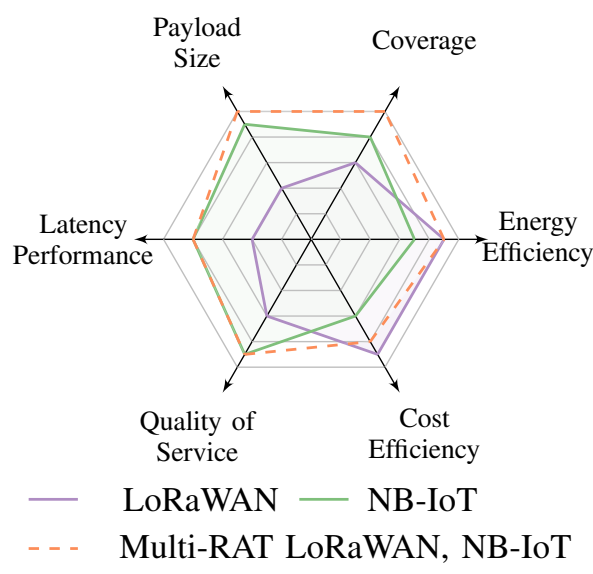

Fig. 1: Comparative study of the explored IoT network technologies, presenting the main IoT feature requirements.

LPWAN communication: providing a relatively small payload size and long-range communication.

For many IoT applications, a single technology suffices. However, use cases under varying operating conditions, may benefit from the integration of multiple wireless technologies. Integration of multiple wireless technologies on a single device can lead to improved performances. Such Multiple Radio Access Technology (Multi-RAT) strategies have previously been used for short-range IoT devices, e.g., incorporating both Wi-Fi and Bluetooth connectivity [3]. Similarly, in cellular networks, different technologies are being used in a fallback strategy. Unlicensed IoT technologies such as Sigfox and LoRaWAN have been combined into a single modem module. However, a true Multi-RAT approach with dynamic switching between both unlicensed and cellular technologies is lacking. Specifically for battery-operated IoT applications, a MultiRAT solution can lead to longer autonomy, since the wireless transmission represents the main energy cost [4]. Based on 
the network conditions or the current needs, e.g., low latency or larger payload size, the IoT device can dynamically switch to the most suitable technology, whilst optimizing for energy efficiency.

This particularly benefits more advanced, dynamic use cases where multiple types of packets need to be sent, or where coverage is not guaranteed. For example, a microphone enabled IoT sensor could send periodic sound level readings using a technology that is most energy efficient for small payloads. When certain events are detected, the device could switch to a connectivity technology that is better suited to transmit a longer sound sample for classification in the cloud. Contributions- The main contribution of this paper is a multiRAT solution that improves on the performance parameters of LPWAN connections, and in particular can prolong the autonomy of battery-powered IoT devices. We focus on the wireless link between the device and the gateway, acknowledging that the operation of the gateways back-end infrastructure will also have an impact on feature requirements such as Quality of Service (QoS) and cost efficiency. In the design of a Multi-RAT solution, the typical requirements and feature set of the IoT technologies are examined. We map these for both LoRaWAN and NB-IoT and provide concrete figures that will be at the basis of the dynamic switching between different LPWAN standards. Although both LoRaWAN and NB-IoT are designed for low-power communication, measurements have shown that large differences in a node's power consumption can be experienced [5].

In [6], the authors have studied the energy consumption of both LoRaWAN and NB-IoT for a smart city application and identify possible opportunities when using Multi-RAT.

The specific contributions of this work are twofold. First, we determine the specification-based boundaries of the most important properties such as coverage, payload size and energy efficiency. The second contribution is the experimental validation of the Multi-RAT potential integrating two LPWAN technologies: LoRaWAN and NB-IoT. We specifically show that for both energy efficiency and latency, a Multi-RAT solution outperforms single technology solutions in scenarios with dynamic payload size, link quality, or QoS requirements. Yet, we also clarify some arguments against deploying a MultiRAT system.

This paper is organized as follows. We determine the theoretical boundaries of a Multi-RAT system, set by the individual LPWAN specifications, exploring both NB-IoT and LoRaWAN. Secondly, our measurements are presented in order to evaluate the energy efficiency and latency in reallife situations. Finally, we summarize our main findings and show how a fitting wireless technology can be selected in a dynamic, Multi-RAT application.

\section{LPWAN MULTI-RAT SUPPORT FOR IOT REQUIREMENTS}

We analyze the main theoretical properties of LoRaWAN and NB-IoT, illustrated in Fig. 1, thereby assessing the potential Multi-RAT opportunities.

\section{A. Energy Efficiency}

Both LoRaWAN and NB-IoT provide measures to minimize energy in support of battery-powered devices. A Multi-RAT system for IoT should select the most energy efficient IoT technology within a set of application parameters (e.g., payload, latency toleration).

LoRaWAN- LoRaWAN is low-power by design. The simple Medium Access Control (MAC) and narrow bandwidth signals contribute to a reduced power consumption. When utilizing LoRaWAN, the energy consumption is mainly determined by the Time on Air (ToA) which depends on (I) the payload size and (II) the Spreading Factor.

One of the advantages of implementing Adaptive Data Rate in the LoRaWAN specification, is optimizing energy efficiency. This algorithm alters the transmit power and data rate of the end-devices depending on the wireless channel conditions. In adverse scenarios, the transmit power and/or spreading factor is increased in order to overcome a low Signal-to-Noise Ratio (SNR). In contrast, when the received SNR is higher than necessary, the device will decrease its transmit power and/or spreading factor.

NB-IoT- To address typical LPWAN-specific requirements, the LTE standard was simplified with an emphasis on energy reduction and lower complexity. Most sleep timers have been extended in the release of NB-IoT in comparison with LTE. In this manner the end-devices can sleep for a longer time period before making contact to the network. The necessity to monitor paging, i.e., listening for down link messages, is drastically reduced by introducing Extended Discontinuous Reception Mode (eDRX). This reduces active radio time, lowering the energy footprint. Long eDRX cycles allow the NB-IoT node to sleep for up to 186 minutes, after which the node checks for paging before going to sleep again.

When a node no longer needs to maintain an active connection, it can go into Power Saving Mode (PSM). In this mode, the node completely disconnects from the network. For a detailed overview of energy consumption in different stages, we refer to Section II-B

\section{B. Coverage}

When deploying IoT nodes in a city landscape or in remote locations, good coverage is critical for obtaining a reliable communication. Both LoRaWAN and NB-IoT feature mechanisms to improve range and coverage. The distance to the nearest gateway clearly is the main determining factor for reliability for both technologies. The networks typically do not share a common infrastructure. Hence, there is inherent redundancy in a Multi-RAT solution and the reliability can be greatly improved with respect to any single RAT IoT connectivity.

LoRaWAN- The range strongly depends on the utilized data rate. Increasing the spreading factor lowers the required demodulation floor and thereby extends the range. The LoRa link budget can be as high as $156 \mathrm{~dB}$ [7] (SF 12).

In contrast to cellular networks, private networks can be deployed in LoRaWAN. Therefore, the coverage can be extended by adding more gateways to the network. 
NB-IoT - Three Coverage Enhancement (CE) levels are baked into NB-IoT. They enable providers to provide network connectivity in places that are hard to reach, such as subterranean parking lots. This way, the Maximum Coupling Loss (MCL) is improved by $20 \mathrm{~dB}$, compared to General Packet Radio Services (GPRS): up to $164 \mathrm{~dB}$. Uplink messages are typically transmitted at $23 \mathrm{dBm}$ [8].

CE levels are decided upon based on the quality of the Radio Frequency (RF) channel, estimated by the Reference Signals Received Power (RSRP): the power of the Reference Signals spread over the full bandwidth. During the network random access procedure, two RSRP thresholds are configured. Therefore, three CE levels can be configured: $\mathrm{CE}$ level 0 (good signal quality), 1 (mediocre signal quality) and 2 (poor signal quality). If there is poor network reception when establishing a connection, the CE level will be increased to level 1 or 2 . High $\mathrm{CE}$ levels result in lower bandwidth and packet repetitions [9]: increasing energy consumption by increased ToA.

T-Mobile [10] performed a measurement campaign to study the distribution of required $\mathrm{CE}$ levels in real-life conditions. No coverage extension was enforced outdoors for $93 \%$ of measurements. For subterranean locations, $\mathrm{CE}$ level 1 is used $27 \%$, while CE level 2 is used $19 \%$ of the time.

\section{Payload Size}

Typical IoT nodes require only a limited amount of bytes to be sent. However, a more generous payload size limit will allow a node to periodically send larger packets. This is particularly useful in surveillance use cases (uplink) and Over The Air (OTA) updates (downlink).

Implementing a Multi-RAT solution provides the combined range of available payload sizes.

LoRaWAN- The payload size defined in LoRaWAN depends on the utilized data rate. For higher spreading factors, LoRaWAN restricts the payload size to 51 bytes, while in more optimal conditions a $242 \mathrm{~B}$ payload can be used in one message (both in uplink and downlink). In case a longer payload is required, the developer has to divide the payload over multiple messages, thereby increasing the reception latency and energy consumption.

NB-IoT - The maximum payload size for each message, regardless of conditions, is $1600 \mathrm{~B}$ [11], both for uplink and downlink messages. There are no duty cycle limits in NB-IoT: an unlimited amount of messages can be sent per day.

\section{Latency}

We consider two latency requirements, i.e. uplink and downlink latency. Uplink latency is defined as the time between the intent of sending a message on the device and receiving that message on the server. This latency, in practice, can be largely attributed to the latency between the IoT node and the gateway. Notably, there is a considerable difference between the intent of sending a message and actual sending the message in license-exempt bands. Downlink latency is defined as latency in the reverse direction.

By characterizing the latency of the implemented technologies, an ideal technology can be selected for latency-critical packets.
As such, the uplink latency is reduced to that of the fastest technology. This emphasises the need for a fine-grained profile of these technologies, as illustrated in this work.

LoRaWAN- The latency of the downlink messages is constrained by the adopted device classes, while the latency of the uplink messages is limited by the duty cycle and data rate.

- Due to license-exempt operation, the devices are subjected to a maximum duty cycle ( $1 \%$ for EU $868 \mathrm{MHz})$. This could increase the latency of uplink messages as they have to respect a minimum wait time before transmitting the next message.

- Three classes of LoRaWAN devices have been defined: A, B and C. The classes vary on different scheduling for down link messages. Class A devices feature two down link windows after each up link message. Class B adds time synchronized receive windows in between up link packets. Class $\mathrm{C}$ devices are constantly able to receive packets as their receive window remains open unless they are transmitting data. Consequently, application requiring low latency downlink messages should adopt class C. Furthermore, the data rate will have an impact on the uplink latency. The data rate of LoRaWAN depends on the utilized SF, bandwidth and Coding Rate (CR). The time on air of a LoRaWAN package, ranges from $25 \mathrm{~ms}$ (SF7, 1 B) to $2.5 \mathrm{~s}$ (SF12, 51 B).

NB-IoT- While uplink latency is limited to $10 \mathrm{~s}$ at most [12] in NB-IoT, downlink latency heavily depends on the set timer values eDRX and PSM.

- Uplink latency is mainly influenced by the path loss and the deployment method of the base station [9]. An extensive NB-IoT latency model has been documented by Azari et al. [13]. When a device needs to send an uplink message, it first needs to listen for cell information. Through this information gathering, the node synchronises with the base station. By sending a Random Access (RA) request to the base station, the device performs access reservation. The base station responds by sending a Random Access Response (RAR), indicating resources reserved for the NB-IoT transfer. Finally, the device is able to send data to the base station.

- Both eDRX timers and PSM timers regulate when the node is able to receive data, thus controlling downlink latency. By prolonging the eDRX cycle, more periodic paging cycles will occur. When in PSM, no packets can be received until the Tracking Area Update (TAU) message is sent.

Typical latency figures reported in literature range from $0.3 \mathrm{~s}$ to $8.3 \mathrm{~s}$, depending on link budget and deployment type [9]. In a stand-alone deployment scenario, full base station power is available to NB-IoT, improving latency. In good coverage conditions, latency is predominantly caused by the time to acquire synchronization and waiting for an access opportunity. Latency in poor coverage conditions are generally caused by latency of the exception report. NB-IoT features a down link data rate of maximal $200 \mathrm{kbps}$ and uplink of maximal $180 \mathrm{kbps}[9]$. 


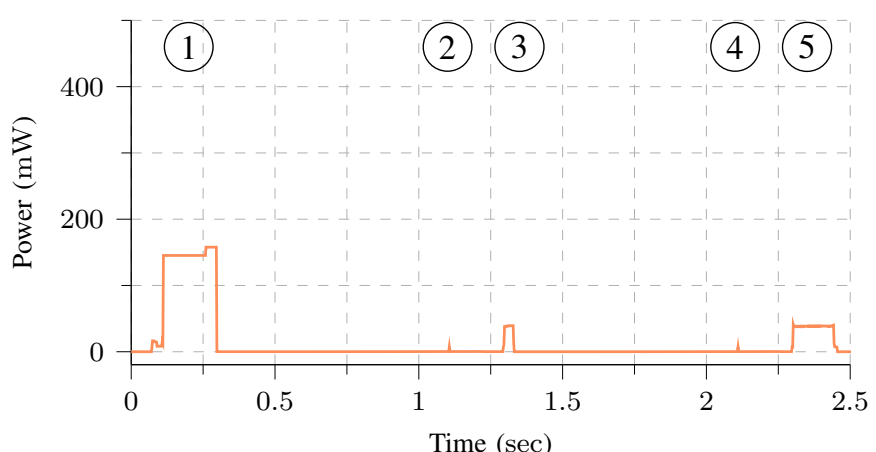

(a) Measured power consumption of a LoRa node (at Spreading Factor (SF) 9): (1) transmit, (2) processing, (3) first receive window, (4) processing, (5) second receive window. Network registration is omitted, as this is not obligatory in LoRaWAN.

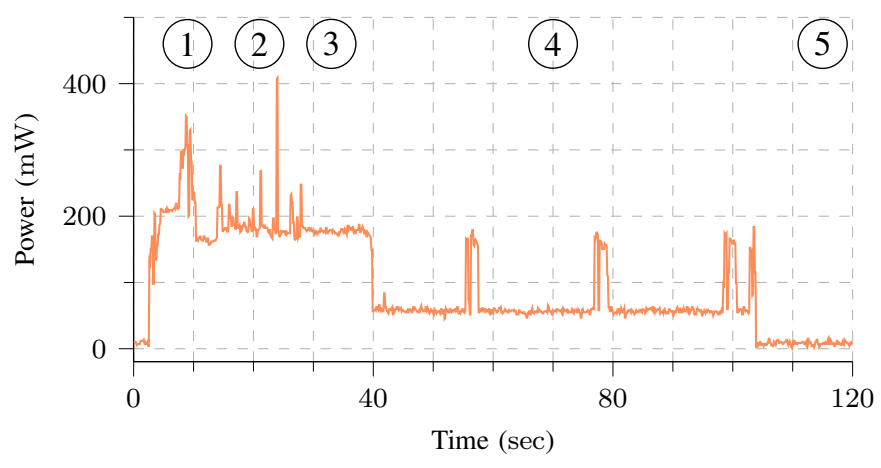

(b) Measured power consumption of a NB-IoT node (at CE level 0): (1) network search and join, (2) package sending, (3) Connected Mode DRX, (4) Extended Discontinuous Reception Mode, (5) Power Saving Mode. Network registration is obligatory and is only performed once (as long as power is maintained).

Fig. 2: Experimentally determined power consumption of LoRa and NB-IoT side by side. Note the large difference in timing, resulting in a larger energy consumption for NB-IoT.

\section{E. Quality of Service}

In IoT-based monitoring use cases, QoS focuses on packet loss and throughput. Mechanisms such as enabling repetitions, increasing output power, etc. allow technologies to control QoS dynamically.

LoRaWAN - The increased interference due to license-exempt operation, is addressed by employing the spread spectrum technique Chirp Spread Spectrum (CSS). LoRaWAN does not support different QoS levels. However, an acknowledgment can be requested so the device can retransmit a message if lost.

NB-IoT- As NB-IoT operates in licensed spectrum, it can offer greater QoS than networks operating in an unlicensed spectrum. Furthermore, NB-IoT employs the same proven time slotted synchronous protocol as used in LTE: ensuring end-toend QoS.

\section{F. Cost Efficiency}

Evidently, the initial hardware investment cost of a Multi-RAT solution will be higher than for a single radio. Several costs need to be considered to get the total cost of ownership: spectrum cost, network deployment cost, the cost of the end device, and eventual costs related to replacement or recharging of batteries involving service visits (relating to energy efficiency). LoRaWAN- As LoRaWAN operates in the unlicensed bands, no spectrum cost is applicable. However, to extend range and network manageability, private networks can be deployed.

NB-IoT-Both the spectrum license and base station contribute significantly to the combined cost of a NB-IoT network, due to NB-IoT running in licensed spectrum. To illustrate, the cost of acquiring the necessary spectrum can amount to $€ 500 \mathrm{M}$ per $\mathrm{MHz}$ [11].

\section{EXPERIMENTAL EVALUATION}

LoRaWAN and NB-IoT have been thoroughly compared and documented in literature; however, experiments-based compar- isons of both energy consumption and latency are lacking. In what follows, these parameters are validated in the field.

\section{A. Experiment Setup}

To evaluate the aforementioned IoT feature requirements, custom hardware has been developed for both LoRa and NBIoT measurements, using commercial off-the-shelf (COTS) electronic components. All files are open source [14 to facilitate reproducibility. In order to assess the technologies in real-life conditions, including the impact of the networkspecific configurations, the Proximus network infrastructure is used as-is.

LoRaWAN- Our LoRaWAN experiments were conducted with a Happy Gecko starter kit, running the LoRaWAN network stack, connected to a LoRa extension module. The board hosts a Semtech SX1272 LoRa Radio chip and runs the LoRaWAN stack. The energy profile of the system is measured with the built-in power monitor of the Happy Gecko.

NB-IoT- The NB-IoT measurements presented in this paper were performed using a custom-made implementation of the Quectel BG96 module. The BG96 is used in a setup which allows us to measure the energy consumption per modem state of the NB-IoT node.

\section{B. Energy Efficiency}

In order to compare the energy efficiency of LoRaWAN and NB-IoT, the energy required to transmit one payload byte $E_{B}$ has been determined. Energy measurements only consider the energy used by the IoT transceiver.

\section{LoRaWAN-}

The measured power profile of a LoRaWAN message is depicted in Fig. 2a In this example a payload of $5 \mathrm{~B}$ is sent with a header of $13 \mathrm{~B}$ (SF 9). The model of [15] combines the measured power consumption in each state and the theoretical air time of a message to compute the total energy consumption of a message. Several states can be observed: transmission of 


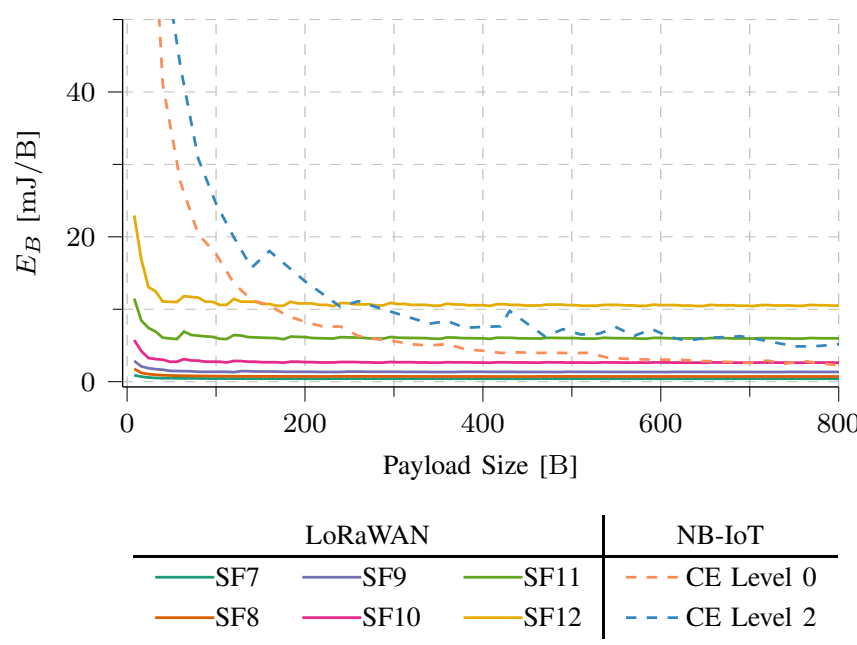

Fig. 3: Energy consumption per byte comparison between LoRaWAN and NB-IoT. The difference in energy consumption is most noticeable when sending small payloads. Over $240 \mathrm{~B}$, NBIoT (CE level 2) is more energy efficient per byte, with respect to LoRaWAN (SF 12).

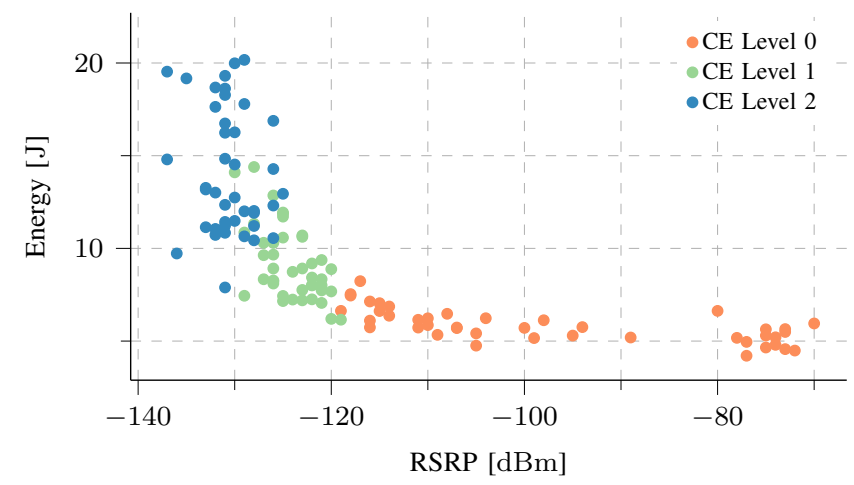

Fig. 4: Energy consumption of an NB-IoT modem by sending a 5-byte payload (18 byte overhead) relative to the observed received power (RSRP). Adapted from [5].

the packet, two processing slots, and two receive windows for receiving downlink messages. Fig. 3 shows the energy per payload byte for different payloads sizes. The payload needs to be divided over multiple messages when the payload size exceeds the maximum message length.

NB-IoT - A typical experimental power consumption measurement is shown in Fig. 2b Several modem states can be observed: network search and join, package send, connected mode Discontinuous Reception Mode (DRX), eDRX and power saving mode.

During the network search and join mechanism, the modem boots and tries to connect to the network. When connecting to the network, the modem negotiates several network parameters (e.g., CE level, timer values, etc.). After the node has successfully joined the network, the package is sent. In this example, five bytes of data encapsulated in a User Datagram Protocol (UDP) packet, resulting in a total NB-IoT-payload of $23 \mathrm{~B}$, is sent. After the message is sent, the modem enters Connected Mode DRX (CDRX). The radio circuitry stays active for a predetermined period, so downlink messages can be received. If eDRX is supported by both the network provider and the NB-IoT node, the modem is able to support periodical downlink communication. To conserve energy, the modem is put to sleep between downlink windows: Paging Time Window (PTW). After a set of eDRX cycles, the modem enters its lowest possible energy state: PSM. Herein, the modem is put to sleep and is not available for any network communication. When the specified PSM timer runs out, the modem reconnects to the network. It is also possible to prematurely reinstate the network communication based on interrupts.

Higher CE levels amount to a larger energy consumption of a NB-IoT node. After measuring the RSRP, the node negotiates the appropriate CE level with the network. This decision directly influences energy consumption for the longevity of the connection. This procedure is experimentally validated in Fig. 4. The total energy consumption is depicted, with respect to the RSRP: the RF signal quality indicator on the NB-IoT node. One can clearly see that a lower RSRP will result in a higher CE level being selected. Moreover, the results clearly indicate that a node transmitting in CE level 2 can use up to four times more energy than a node transmitting in CE level 0 . NB-IoT supports a maximum of $1600 \mathrm{~B}$ to be sent in a single packet. The effective payload size also influences the energy efficiency of NB-IoT. As fixed energy costs, such as CDRX and eDRX, are equal for smaller and larger payload sizes, the energy per byte $\left(E_{B}\right)$ will be lower for longer packets (Fig. 3). Notably, NB-IoT (at CE level 2) is more energy efficient than LoRaWAN (at SF 12) when the payload size exceeds $240 \mathrm{~B}$. For payloads larger than $240 \mathrm{~B}$, NB-IoT becomes progressively and up to 7 times more energy efficient than LoRaWAN with larger payloads. The energy impact of rising $\mathrm{CE}$ levels, however, is reduced when transmitting large payloads.

\section{Latency}

Latency in LoRaWAN and NB-IoT was evaluated, hereby focusing on uplink latency.

LoRaWAN- The uplink latency is limited by the allowed air time or, equivalently, duty cycle. The uplink latency for payloads sizes of 1600 bytes ranges from $42 \mathrm{~min}$ to $68 \mathrm{~h}$ (only the default and mandatory bands are used, i.e., $1 \%$ duty cycle). As the maximum message size is constrained by the spreading factor, the payload needs to be split up in multiple messages in order to send the full payload, increasing the uplink latency. The total duty cycle is limited by the supported operating bands of the network.

NB-IoT- According to NB-IoT specifications, the latency should be kept under $10 \mathrm{~s}$. This was experimentally validated by sending 1236 packages in varying signal conditions. The results, depicted in Fig. 5, show that both CE levels 0 and 1 keep latency within the $10 \mathrm{~s}$ maximum. Packets transmitted in CE level 1 only have a slightly larger latency than packets transmitted in CE level 0. When transmitting on CE level 2, however, poor signal conditions can cause latency to reach up to $20 \mathrm{~s}$. The median latency increases and more extreme latency outliners occur. In NB-IoT, latency is barely influenced by payload size. 


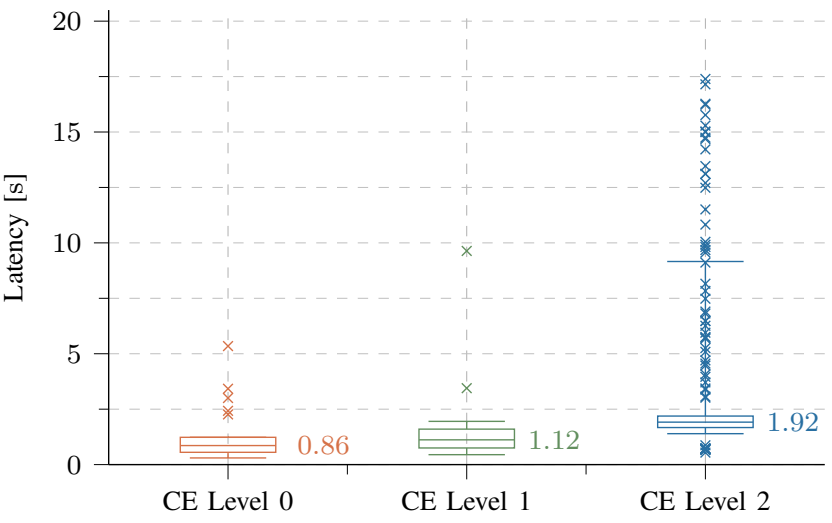

Fig. 5: Boxplot of measured NB-IoT latency when sending a packet: $14 \mathrm{~B}$ payload, CE level 2.

\section{CONCLUSIONS}

Considering the predominant requirements for an LPWAN node, our assessment has highlighted the complementarities and potential synergies of LoRaWAN and NB-IoT technologies. While for many basic IoT applications this leads to one preferred technology, implementing a Multi-RAT scheme in more dynamic use cases can greatly improve a node's functionality and energy efficiency. Based on our study and experimental validation, we demonstrate the potential of a Multi-RAT (LoRaWAN/NB-IoT) solution from the perspective of different IoT requirements, while focusing on an optimal energy trade-off.

\section{A. Potential Multi-RAT Gains}

a) Energy Efficient Operation for Variable Payload Sizes: IoT use cases with varying payload sizes benefit from a MultiRAT approach. By implementing NB-IoT, messages up to $1600 \mathrm{~B}$ can be sent, while still enabling extremely low-power messages with small payloads over LoRaWAN. Considering the case of smart city surveillance, whereby a node would send hourly sensor data (e.g., sound level), and more extensive data when certain thresholds are met (e.g. loud noises). The Multi-RAT solution would select LoRaWAN for hourly sensor updates (based on existing use cases [15]: 16 B, 97\% of packages), and NB-IoT for sending more elaborate sensor recordings for classification (assumed $1600 \mathrm{~B}$, occurring for example 5 times per week or $3 \%$ of sent messages). This would result in $23 \mathrm{~J}$ transmit energy per week, a reduction of times 15 with respect to only using NB-IoT and of times 4 when only using LoRaWAN. To make this more tangible, this would mean that the device could, in ideal conditions, operate for 30 years on a single smartphone battery (2500 mAh). LoRaWAN would require the large payloads being split into 32 messages, which would clearly also introduce a latency penalty.

b) Guaranteeing Timely Delivery for Latency-Critical Messages: Depending on network coverage and network load, latency can be optimized by spreading communications over multiple IoT technologies. When low energy consumption is critical and the NB-IoT chipset is in PSM, a faster wake-up can be achieved with LoRaWAN. On the other hand, larger payloads can be sent more rapidly with NB-IoT. This could be important for monitoring medical grade parameters (e.g., heart rhythm or fall detection).

c) Redundant Networking and Improving Service Area: By combining multiple IoT technologies, the effective service area of a Multi-RAT solution will be extended to the area of all IoT technologies on-board. When coverage is not provided by one IoT technology, another can step in. The service area can be privately extended for LoRaWAN by deploying private gateways.

d) Improving QoS: By enabling an IoT device to operate on both LoRaWAN and NB-IoT, the optimal QoS for any message can be chosen: increasing robustness and reliability. Periodic 'alive' messages do not need high QoS, yet more important messages containing sensitive data (e.g., temperature tracking on track and trace applications) do need high QoS. LoRaWAN should be used for the periodic, low QoS, messages, saving energy. Important messages can be sent through the NB-IoT network: featuring high QoS.

\section{B. Potential Multi-RAT Drawbacks}

a) Device Footprint: By including multiple IoT technologies on one device, multiple modems will need to be on-board. This increases the space needed for wireless interfaces by both the applicable modem size and the appropriate antenna size. The board space occupied by multiple modems, can be improved by efficiently using both sides of a Printed Circuit Board (PCB). Multiple antennas can be combined in multiband antennas.

b) Device Cost: As multiple modems are included, hardware and network subscription costs will rise. However, by optimizing energy consumption, costs can be saved by not requiring manual intervention for battery replacements.

c) Computational Overhead: To dynamically switch between IoT technologies, some computational overhead will be required. The energy savings from implementing a Multi-RAT platform, however, the energy savings of Multi-RAT outweighs the energy consumption of the required additional computations. [4].

\section{Conclusions}

In many cases, the prioritization of strict IoT requirements is not an easy feat. For example, when an application requires both small and larger data transfers, the choice of IoT technology will affect the energy consumption, latency, etc., of both types of messages. The Multi-RAT solution presented in this paper allows to dynamically adapt the stated IoT properties priorities (and thus IoT technology), depending on the most prominent IoT requirement and current context. This in particular benefits energy consumption that can be reduced by impressive factors. The gathered empirical data can help to select either the most appropriate connectivity solution and contribute to a behavioural model of the latency and power consumption, used to develop Multi-RAT dynamic operation procedures: optimizing efficiency and effectivity. 


\section{REFERENCES}

[1] K. Mekki, E. Bajic, F. Chaxel, and F. Meyer, "Overview of Cellular LPWAN Technologies for IoT Deployment: Sigfox, LoRaWAN, and NB-IoT," in 2018 IEEE PerCom. IEEE, 2018, pp. 197-202.

[2] R. Balani, "Energy Consumption Analysis for Bluetooth, Wifi and Cellular Networks," Tech. Rep. TR-UCLA-NESL-200712-01, 2007.

[3] W. Lee, J. Koo, Y. Park, and S. Choi, "Transfer time, energy, and quotaaware multi-rat operation scheme in smartphone," IEEE Transactions on Vehicular Technology, vol. 65, no. 1, pp. 307-317, 2015.

[4] H. Karl and A. Willig, Protocols and Architectures for Wireless Sensor Networks. John Wiley \& Sons, 2007.

[5] G. Leenders, G. Callebaut, L. Van der Perre, and L. De Strycker, "An Experimental Evaluation of Energy Trade-Offs in Narrowband IoT," in 2020 IEEE 6th WF-IoT. IEEE, 2020.

[6] K. Mikhaylov, M. Stusek, P. Masek, V. Petrov, J. Petajajarvi, S. Andreev, J. Pokorny, J. Hosek, A. Pouttu, and Y. Koucheryavy, "Multi-Rat LPWAN in smart cities: Trial of LoRaWan and NB-IoT Integration," in 2018 IEEE ICC. IEEE, 2018, pp. 1-6.

[7] A. J. Wixted, P. Kinnaird, H. Larijani, A. Tait, A. Ahmadinia, and N. Strachan, "Evaluation of LoRa and LoRaWAN for Wireless Sensor Networks," in 2016 IEEE SENSORS. IEEE, 2016, pp. 1-3.

[8] P. Andres-Maldonado, P. Ameigeiras, J. Prados-Garzon, J. NavarroOrtiz, and J. M. Lopez-Soler, "Narrowband IoT Data Transmission Procedures for Massive Machine-Type Communications," IEEE Network, vol. 31, no. 6, pp. 8-15, 2017.

[9] O. Liberg, M. Sundberg, E. Wang, J. Bergman, and J. Sachs, Cellular Internet of Things: Technologies, Standards, and Performance. Academic Press, 2017.

[10] "Narrowband IoT Delivers Insights from the larges NB-IoT Indoor Measurement Campaign," Deutsche Telekom AG, Tech. Rep., January 2019.

[11] K. Mekki, E. Bajic, F. Chaxel, and F. Meyer, "A Comparative Study of LPWAN Technologies for Large-Scale IoT Deployment," ICT express, 2019.

[12] R. Ratasuk, N. Mangalvedhe, Y. Zhang, M. Robert, and J.-P. Koskinen, "Overview of Narrowband IoT in LTE Rel-13," in 2016 IEEE CSCN. IEEE, 2016.

[13] A. Azari, G. Miao, C. Stefanovic, and P. Popovski, "Latency-Energy Tradeoff Based on Channel Scheduling and Repetitions in NB-IoT Systems," in 2018 IEEE GLOBECOM. IEEE, 2018.

[14] G. Leenders, "Multi RAT for IoT - Source files." [Online]. Available: dx.doi.org/10.5281/zenodo.5069274 [Accessed on: July 1, 2021].

[15] G. Callebaut, G. Leenders, J. Van Mulders, G. Ottoy, L. De Strycker, and L. Van der Perre, "The art of designing remote iot devices-technologies and strategies for a long battery life," Sensors, vol. 21, no. 3, 2021.
Guus Leenders Guus Leenders received his master's degree summa cum laude in engineering technology at KU Leuven in 2015. He is member of the Dramco research group. There, he is involved with numerous projects in IoT. His interests are Internet of Things and embedded systems.

Gilles Callebaut Gilles graduated summa cum laude in 2016 and received the M.Sc. degree in engineering technology at KU Leuven. He is currently a member of the Dramco reserach group. His interests are Machine Type Communication, Internet of Things, embedded systems and everything mobile.

Geoffrey Ottoy Geoffrey is a researcher on Internet of Things applications. In 2013 he received his Ph.D. in Electrical Engineering from KU Leuven. His interests are indoor localization and low-power embedded systems.

Liesbet Van der Perre Liesbet Van der Perre received her $\mathrm{PhD}$ degree in Electrical Engineering from KU Leuven, Belgium. She is professor at Dramco, KU Leuven. Her research interest are energy efficient wireless communication and embedded systems.

Lieven De Strycker Lieven De Strycker is professor at the Faculty of Engineering Technology, Department of Electrical Engineering, KU Leuven. He joined the Engineering Technology department of the Catholic University College Ghent, where he founded the Dramco research group at KU Leuven. 


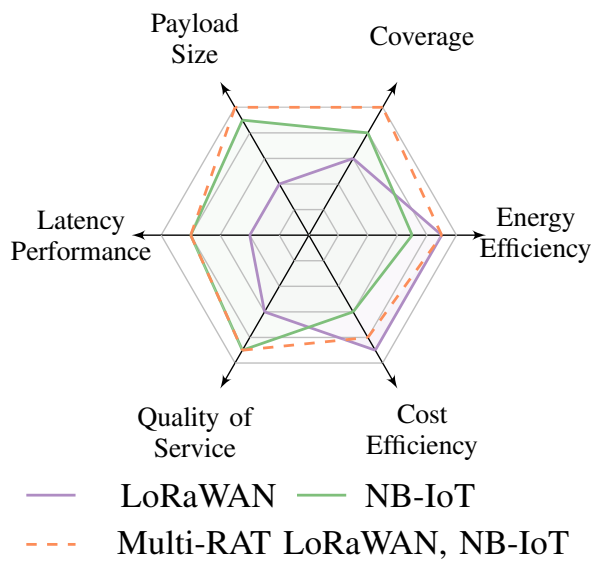

Fig. 1: Comparative study of the explored IoT network technologies, presenting the main IoT feature requirements. 


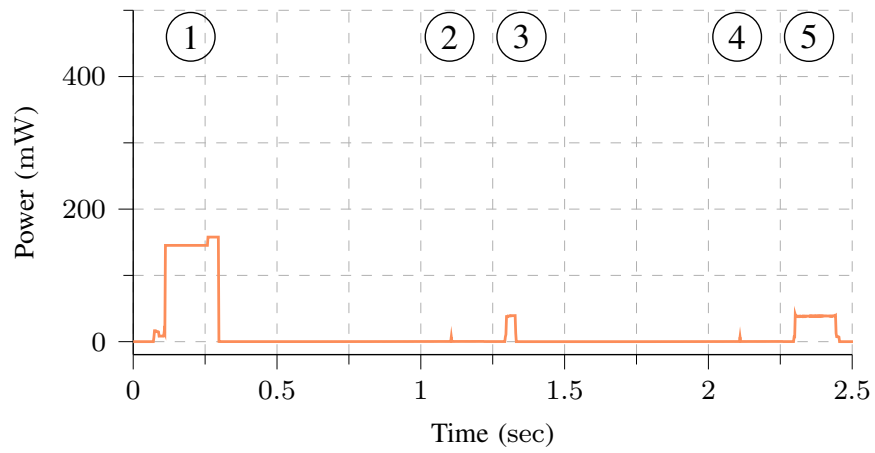

(a) Measured power consumption of a LoRa node (at SF 9): (1) transmit, (2) processing, (3) first receive window, (4) processing, (5) second receive window. Network registration is omitted, as this is not obligatory in LoRaWAN.

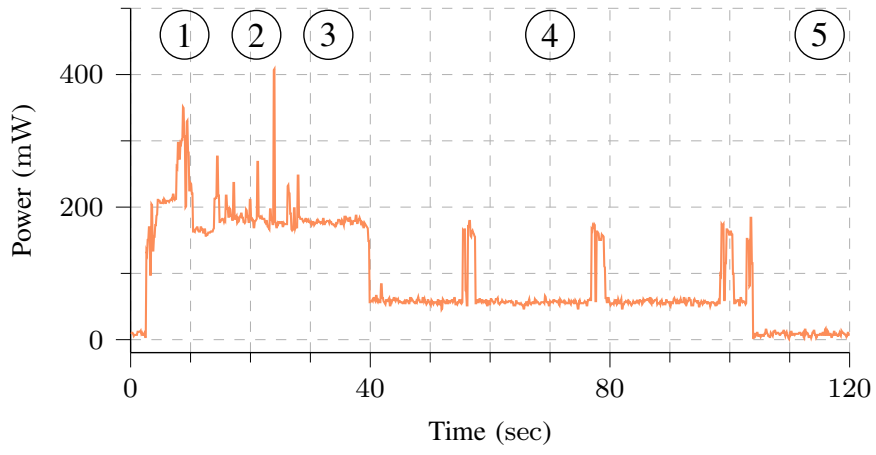

(b) Measured power consumption of a NB-IoT node (at CE level 0): (1) network search and join, (2) package sending, (3) Connected Mode DRX, (4) Extended Discontinuous Reception Mode, (5) Power Saving Mode. Network registration is obligatory and is only performed once (as long as power is maintained).

Fig. 2: Experimentally determined power consumption of LoRa and NB-IoT side by side. Note the large difference in timing, resulting in a larger energy consumption for NB-IoT. 


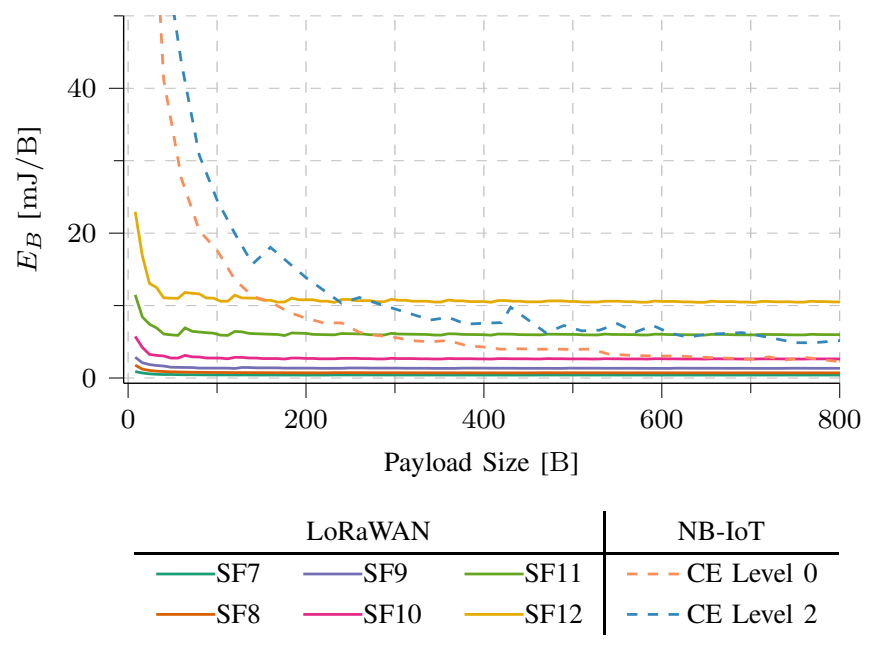

Fig. 3: Energy consumption per byte comparison between LoRaWAN and NB-IoT. The difference in energy consumption is most noticeable when sending small payloads. Over $240 \mathrm{~B}$, NBIoT (CE level 2) is more energy efficient per byte, with respect to LoRaWAN (SF 12). 


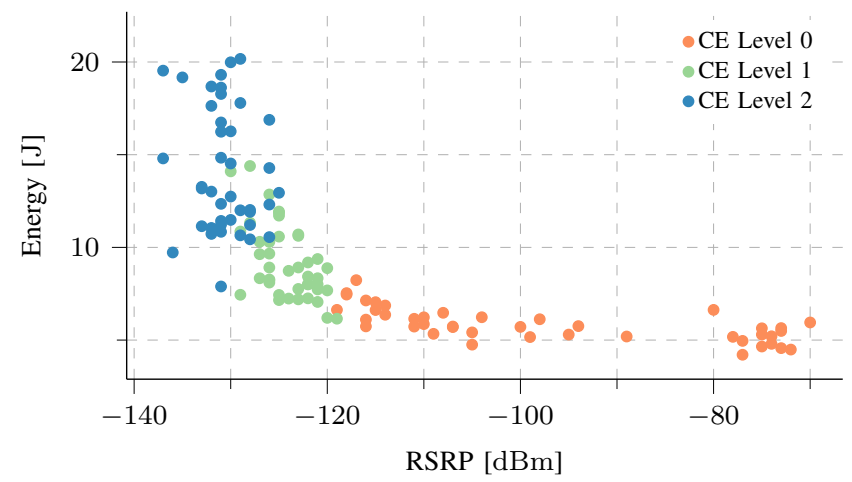

Fig. 4: Energy consumption of an NB-IoT modem by sending a 5-byte payload (18 byte overhead) relative to the observed received power (RSRP). Adapted from [5]. 


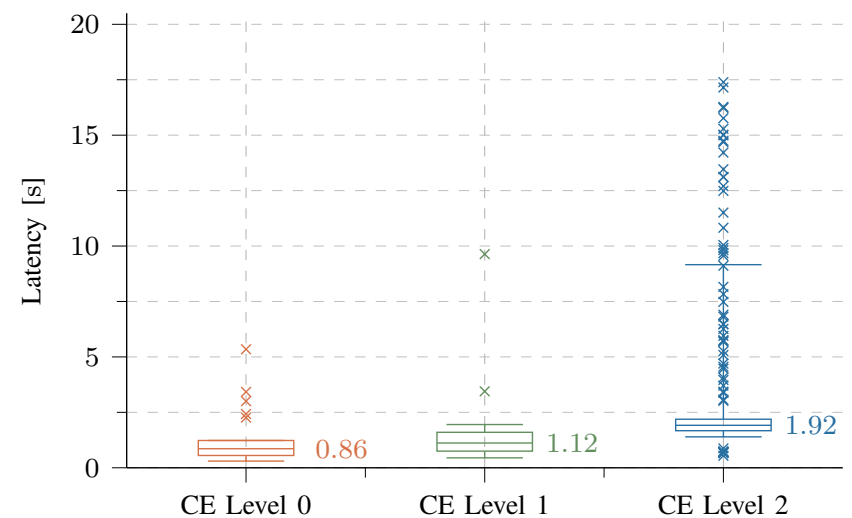

Fig. 5: Boxplot of measured NB-IoT latency when sending a packet: $14 \mathrm{~B}$ payload, CE level 2. 